\title{
High resolution limb images synthesized from 3D MHD simulations
}

\author{
Mats Carlsson ${ }^{1} \dagger$ \\ Robert F. Stein ${ }^{2}$ \\ Åke Nordlund ${ }^{3}$ \\ and Göran B. Scharmer ${ }^{4}$ \\ ${ }^{1}$ Institute of Theoretical Astrophysics, University of Oslo, Norway \\ ${ }^{2}$ Dept. of Physics and Astronomy, Michigan State University, East Lansing, USA \\ ${ }^{3}$ NBIfAFG, University of Copenhagen, Denmark \\ ${ }^{4}$ The Institute for Solar Physics of the Royal Swedish Academy of Sciences, Stockholm, \\ Sweden
}

\begin{abstract}
We present the first center-to-limb G-band images synthesized from high resolution simulations of solar magneto-convection. Towards the limb the simulations show "hilly" granulation with dark bands on the far side, bright granulation walls and striated faculae, similar to observations. At disk center G-band bright points are flanked by dark lanes. The increased brightness in magnetic elements is due to their lower density compared with the surrounding intergranular medium. One thus sees deeper layers where the temperature is higher. At a given geometric height, the magnetic elements are cooler than the surrounding medium. In the Gband, the contrast is further increased by the destruction of $\mathrm{CH}$ in the low density magnetic elements. The optical depth unity surface is very corrugated. Bright granules have their continuum optical depth unity $80 \mathrm{~km}$ above the mean surface, the magnetic elements $200-300 \mathrm{~km}$ below. The horizontal temperature gradient is especially large next to flux concentrations. When viewed at an angle, the deep magnetic elements optical surface is hidden by the granules and the bright points are no longer visible, except where the "magnetic valleys" are aligned with the line of sight. Towards the limb, the low density in the strong magnetic elements causes unit line-of-sight optical depth to occur deeper in the granule walls behind than for rays not going through magnetic elements and variations in the field strength produce a striated appearance in the bright granule walls.
\end{abstract}

3D magneto-convection simulations

- non-grey, LTE radiation in energy balance

- $6 \times 6 \mathrm{Mm}, 1 \mathrm{Mm}$ above, $2.5 \mathrm{Mm}$ below photosphere

- 253x253x163 points, $25 \mathrm{~km}$ horizontal res. $15-35 \mathrm{~km}$ vertically.

- start from uniform vertical magnetic field of $250 \mathrm{G}$

Spectrum synthesis:

- 2 bands $3 \mathrm{~nm}$ wide, 1364 wavelength points

○ $430.68 \mathrm{~nm}$ (G-band)

○ $436.52 \mathrm{~nm}$ (G-continuum)

- 1838 lines, depth dependent Voigt profiles

- filter functions from Swedish 1m Solar Telescope

$\dagger$ Also at Center of Mathematics for Applications, University of Oslo, P.O. Box 1053 Blindern, N-0316 Oslo, Norway. 
Gcont $\mu=0.4$

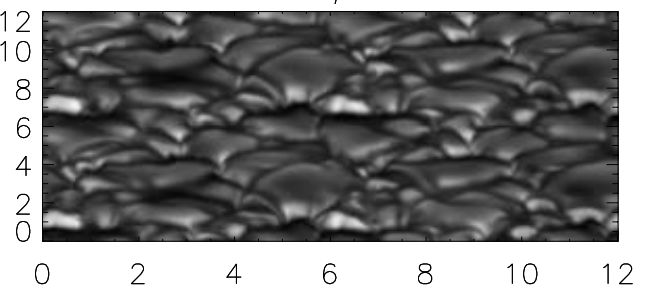

Gcont $\mu=0.6$
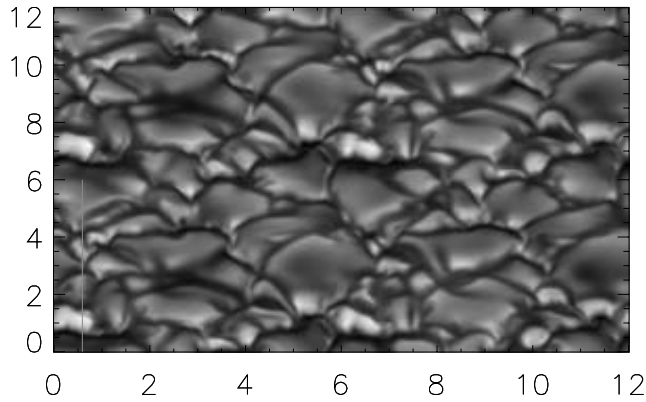

Gont $\mu=0.8$
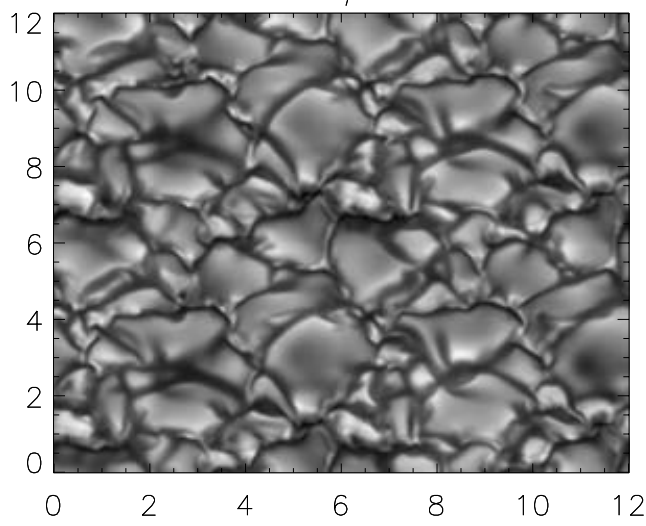

Gcont $\mu=1.0$

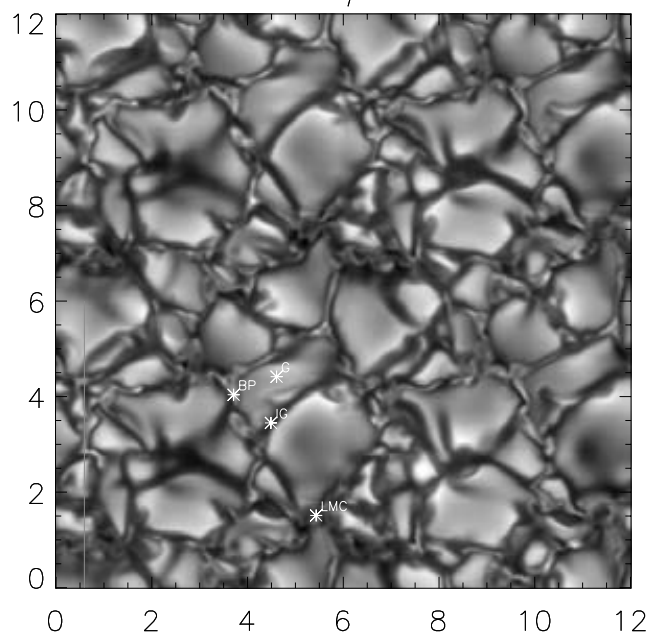

Gband $\mu=0.4$

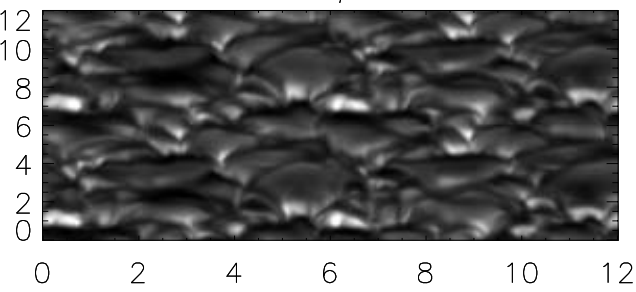

Gband $\mu=0.6$

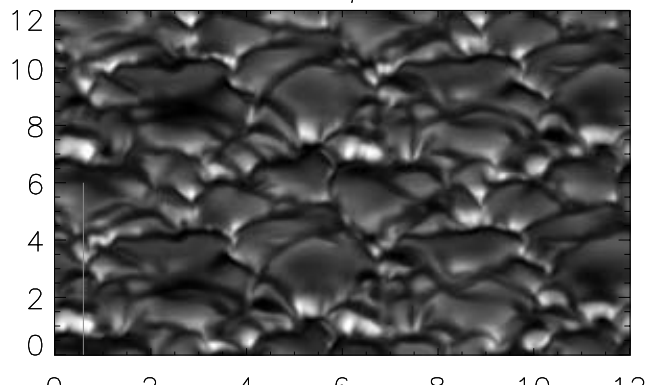

Gband $\mu=0.8$
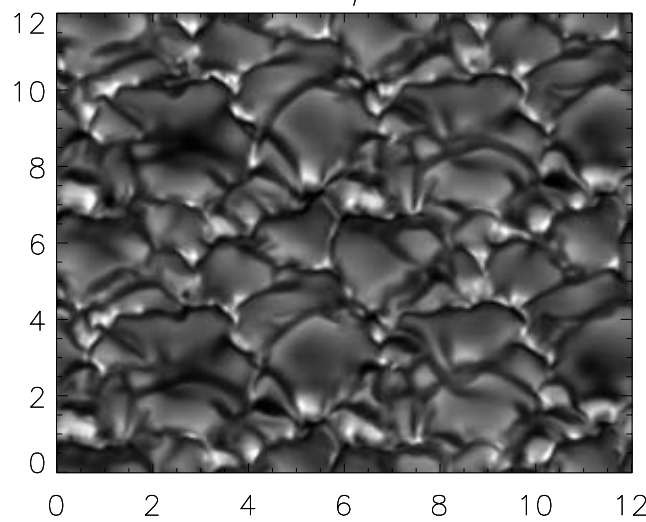

Gband $\mu=1.0$

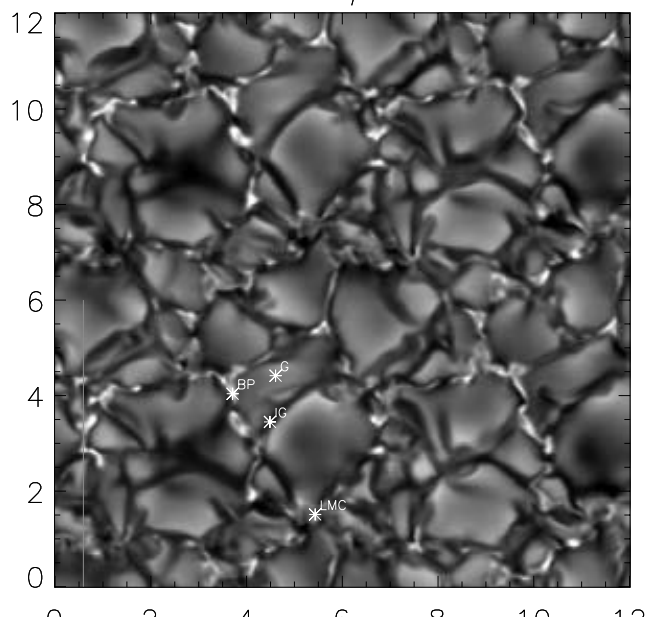

Figure 1. G-cont and G-band images at disk center and $\mu=0.8,0.6,0.4$. Axes are distances in $\mathrm{Mm}$. The panels have individual scalings. Four snapshots one minute apart are shown in each frame in order upper-left,lower-left,upper-right,lower-right. 